\title{
Factores sociodemográficos asociados a la actividad física deportiva en adolescentes mexicanos
}

\section{Sociodemographic factors associated with sports-related physical activity in Mexican adolescents}

\section{AUTORES \\ Carolina Ayala Hilario ${ }^{1}$ (D) \\ César Ayala-Guzmán ${ }^{1}$ (D) \\ Luis Ortiz-Hernández ${ }^{1}$ (D) \\ 1 Universidad Autónoma Metropolitana Unidad Xochimilco. Departamento de Atención a la Salud. Ciudad de México, México}

\section{CONTACTO}

Luis Ortiz-Hernández

E-mail:lortiz@correo.xoc.uam.mx

Calz. del Hueso 1100, Col. Villa Quietud,

Coyoacán, Ciudad de México, México.

CP: 04960 .

DOI

10.12820/rbafs.24e0093

\section{(cc) BY-NC-SA}

Este obra está licenciado com uma Licença Creative Commons Atribuição-NãoComercialCompartilhaI gual 4.0 Internacional.

\begin{abstract}
RESUMEN
El objetivo del estudio fue analizar los cambios en la prevalencia de actividad física deportiva (AFD) en adolescentes mexicanos y la asociación de ésta con variables sociodemográficas y la AFD de sus padres o madres. Se trata de un estudio transversal y comparativo en el que se analizó la Encuesta Nacional de Uso del tiempo (ENUT) versiones 2002 y 2014, la cual se basa en una muestra representativa de toda la población mexicana con 12 años o más. Se seleccionó la información de adolescentes de 12 a 19 años $(n=11,795)$ y de sus padres $(n=8,161)$ o madres $(n=10,261)$. Por medio de un cuestionario se indagó sobre características sociodemográficas, la práctica de AFD y el tiempo destinado a ésta. Se estimaron frecuencias, estadísticos de $\mathrm{Chi}^{2}$, T de Student y modelos de regresión logística. En doce años la prevalencia de AFD de adolescentes aumentó de 44.0\% a 55.6\% $(\mathrm{p}<0.001)$, aunque no cambió el tiempo destinado a realizarla $(\mathrm{p}=0.250)$. Los adolescentes que eran mujeres, que tenían mayor edad, con padres y/o madres con menor escolaridad y que éstos no practicaran AFD tuvieron menor probabilidad de realizar AFD. La AFD reportada por los adolescentes se relacionó más con la AFD de sus padres que con la AFD de las madres. A pesar de que en 12 años aumentó la práctica de AFD reportada por adolescentes mexicanos, existen grupos con baja probabilidad de realizarla, en los cuales se pueden enfocar políticas y programas públicos diseñados a la promoción de estilos de vida activos.
\end{abstract}

Palavras clave: Actividad motora; Ejercicio; México; Adolescentes.

\begin{abstract}
The aim of this study was to analyze changes in the prevalence of sports-related physical activity (SPA) in Mexican adolescents and its association with sociodemographic variables and the SPA of their parents. This is a cross-sectional and comparative study in which it was analyzed the National Survey of Use of Time (ENUT) versions 2002 and 2014, which is based on a representative sample of the entire Mexicans population over 12 years of age. The information of adolescents aged 12 to 19 years $(n=11,795)$ was selected, as well as that of their fathers $(n=8,161)$ or mothers $(n=10,261)$. Through a questionnaire, it was inquired about sociodemographic characteristics, SPA and the time allocated to perform it. Frequencies, Chi-squared, and Student T statistics and logistic regression models were estimated. Over a period of 12 years the prevalence of adolescent's reported SPA increased from $44.0 \%$ to $55.6 \%(p<0.001)$, although there was no change in the reported time spent in this activity $(p=0.250)$. Adolescents who were girls, who were older, with parents and/or mothers with less schooling and those with parents who did not perform SPA were more likely that did not practice SPA. Reported SPA of adolescents was more related to fathers' SPA than with the mothers' SPA. Although the practice of SPA reported by Mexican adolescents increased in 12 years, there are groups with a low probability of performing it, in which policies and public programs designed to promote active lifestyles can be focused.
\end{abstract}

Keywords: Motor activity; Exercise; Mexico; Adolescent.

\section{Introducción}

La inactividad física en población pediátrica está asociada con mayor adiposidad, presión arterial, lípidos en sangre y hemoglobina glucosilada; y menor condición física. Además, la práctica de actividad física (AF) y los comportamientos sedentarios en etapas tempranas tienden a continuar hasta la edad adulta ${ }^{1}$.

La AF puede clasificarse en actividades obligatorias (i.e. ocupacionales, para transporte, domésticas, asis- tencia a la escuela) y discrecionales (i.e. actividades dirigidas directamente a mejorar las condiciones de salud, a la recreación y socialización) ${ }^{2}$.La principal actividad obligatoria de adolescentes es la asistencia a la escuela, lo cual involucra dedicar una cantidad sustancial de tiempo a estar sentado. Por ello en esta edad, la principal forma de $\mathrm{AF}$ es la que se asocia con actividades discrecionales como juego o deporte. Esto es relevante ya que la actividad física moderada y vigorosa (AFMV) 
y la actividad física deportiva (AFD) se relacionan con la masa corporal ${ }^{3}$, de las cuales, la AFD tiene mayor influencia en el peso corporal de adolescentes ${ }^{4}$.

La escasa evidencia disponible señala que en países de ingreso alto ha aumentado la AFD de adolescentes ${ }^{5}$. En contraste, en países de ingreso medio, pocos estudios han analizado la evolución de la $\mathrm{AF}$ en general y de la AFD en específico. En México, existen estimaciones de la prevalencia de AFMV en población pediátrica ${ }^{6}$, pero es escasa la información sobre los cambios de ésta a través del tiempo, así como de los factores sociodemográficos que pueden influir en su práctica. En la Encuesta Nacional de Salud y Nutrición (ENSANUT) se ha analizado y comparado los cambios en la prevalencia de la AF de la población pediátrica mexicana de 2002 a $2016^{6}$, considerando como físicamente activos a los adolescentes que refirieron realizar $>420$ minutos de AFMV a la semana. Además, únicamente se ha reportado la prevalencia de AFMV en la población total y de acuerdo con el sexo de los adolescentes, pero falta conocer la influencia de factores sociodemográficos en la activación física de adolescentes y el tiempo destinado a ésta.

Es escasa la información respecto a la influencia de la familia y amigos en la práctica de AF de niños y adolescentes ${ }^{7}$. Pocos estudios han analizado las asociaciones entre la práctica de AF con características sociodemográficas de la población pediátrica ${ }^{8}$ o de sus padres o madres ${ }^{9}$. Se ha reportado que la población pediátrica de menor nivel socioeconómico tiene menor probabilidad de ser físicamente activa ${ }^{10}$, que existen diferencias en la práctica de $\mathrm{AF}$ de acuerdo con el sexo ${ }^{11}$ y que la $\mathrm{AF}$ se asocia con la edad ${ }^{12}$; sin embargo, sigue siendo escasa la información sobre la influencia de factores sociodemográficos y de la AFD de padres o madres en la práctica de AFD de población pediátrica, principalmente mexicana.

Conocer la prevalencia de AFD en la población en general e identificar grupos con menor probabilidad de realizarla podría ayudar al diseño de políticas y programas encaminados a su promoción. Además, documentar los cambios en las prevalencias a lo largo del tiempo permite conocer la evolución de las desigualdades sociales relacionadas con esta conducta. $\mathrm{El}$ primer objetivo de este estudio fue conocer los cambios en la prevalencia de AFD en adolescentes mexicanos entre 2002 y 2014. El segundo objetivo fue analizar la relación de características sociodemográficas de los adolescentes y de sus padres y/o madres con la práctica de AFD de los mismos.

\section{Métodos}

Se trata de un estudio transversal y comparativo en el que se analizaron las bases de datos de la Encuesta Nacional de Uso del Tiempo (ENUT) versiones 2002 y 2014, que es representativa del territorio nacional y realizada por el Instituto Nacional de Estadística y Geografía $^{13}$. El trabajo de campo de la ENUT 2002 se realizó en noviembre y diciembre y el de la ENUT 2014 en octubre y noviembre. La ENUT se basa en un muestreo probabilístico, bi-etápico, estratificado y por conglomerados. Las muestras totales de habitantes de las viviendas fueron de 20342 en 2002 y 42118 en 2014. La tasa de no respuesta fue de $25.2 \%$ en 2002 y $10.21 \%$ en 2014. En 2014 se entrevistaron a 8493 adolescentes de los cuales 8245 respondieron la pregunta sobre AFD. La base de datos del 2002 contenía información completa de 3550 adolescentes. Por lo tanto, se consideró la información de 11795 adolescentes de 12 a 19 años que respondieron la pregunta sobre AFD, y de sus padres (2588 en 2002 y 5573 en 2014) o madres (3213 en 2002 y 7048 en 2014). La información se obtuvo por medio de un cuestionario aplicado por encuestadores entrenados.

La ENUT es parte del sistema de información gubernamental; por lo tanto, la participación en la encuesta es obligatoria ${ }^{13}$. Durante el trabajo de campo se obtiene el consentimiento informado verbal de los participantes y se garantiza la confidencialidad de la información. Por ello, las bases de datos no contienen datos que permitan identificar a los participantes. La aprobación ética del análisis de la ENUT aquí reportado fue otorgada por el Comité de Ética de la División de Ciencias Biológicas y de la Salud de la Universidad Autónoma Metropolitana de Xochimilco.

Las variables independientes fueron: escolaridad de los padres y/o madres, tamaño de localidad y características sociodemográficas del encuestado. La escolaridad de los padres y/o madres fue utilizada como un indicador del nivel socioeconómico y fue clasificada en cinco categorías: ninguna, primaria, secundaria, preparatoria y superior (incluyendo superior y posgrado). E1 tamaño de la localidad se clasificó en cuatro categorías: ciudades ( $\geq 100000$ habitantes), urbanas (15000 a 99999 habitantes), mixtas (2500 a 14999 habitantes) y rurales (< 2500 habitantes). Las características demográficas de los participantes que se analizaron fueron la edad y el sexo.

En la ENUT se indaga si los adolescentes y sus padres/madres realizaron AFD en la semana previa a la 
encuesta. La pregunta en el 2002 fue: “¿En la misma semana hizo ejercicio, deporte o jugó? (no incluir si es por trabajo o estudio)", mientras que en 2014 fue: “Durante la semana pasada, en su tiempo libre ¿usted hizo deporte o ejercicio físico? (fútbol, basquetbol, natación, box, karate, caminar, correr, patinar, andar en bicicleta, yoga, zumba)". En esta pregunta se considera el deporte y ejercicio como sinónimos, aunque no lo sean, por lo que nos referimos a este comportamiento como AFD. Si la respuesta era positiva, se indagaba sobre el tiempo entre semana y en fin de semana que se destinaba a ella.

Para el análisis estadístico se utilizó el programa STATA versión 14. Las estimaciones se hicieron utilizando los comandos de encuestas (svy), con lo cual se consideró el diseño complejo de la ENUT. Se obtuvieron las frecuencias absolutas y relativas de las variables bajo estudio (tabla 1,2 y 3 ). Cuando se hicieron comparaciones entre grupos se utilizó estadística $\mathrm{Chi}^{2}$ para variables categóricas y la prueba de $\mathrm{T}$ de student para muestras independientes para variables continuas. Posteriormente, se estimaron modelos de regresión logística, en los cuales la variable dependiente fue la realización de AFD por los adolescentes; mientras que las variables independientes fueron las características sociodemográficas del encuestado. Al realizar el análisis de los datos se observó que una proporción importante de adolescentes no vivía con sus padres y madres (19.2\% en 2002 y $24.8 \%$ en 2014). Por lo tanto, se realizaron 3 modelos de regresión logística. En el modelo 1 se muestran las probabilidades (razón de momios, $\mathrm{RM}$ ) de realizar AFD de adolescentes que únicamente vivían con sus padres, en el modelo 2 con aquellos que vivían con sus madres, en el modelo 3 con aquellos que vivían con sus padres y madres. Los tres modelos fueron ajustados por el año de la encuesta, por el sexo y edad de los adolescentes, por la edad y AFD de los padres. En los modelos se incluyeron todas las interacciones posibles del año de la encuesta con el resto de las covariables (i.e. características de los adolescentes, sus padres/madres). Cuando existieron interacciones significativas $(p<0.100)$, se estimaron y graficaron las probabilidades ajustadas de realizar AFD por los adolescentes (Figura 1). Cuando las interacciones no eran significativas se descartaron de los modelos.

\section{Resultados}

Entre ambos años no existieron diferencias en la distribución de acuerdo con el sexo y edad de los adolescentes (Tabla 1). Sin embargo, en el transcurso de doce años
Tabla 1 - Características sociodemográficas y de práctica de actividad física deportiva en adolescentes mexicanos en 2002 y 2014

\begin{tabular}{|c|c|c|c|}
\hline & 2002 & 2014 & \multirow{2}{*}{$\mathrm{p}$} \\
\hline & $\%(\mathrm{n})$ & $\%(\mathrm{n})$ & \\
\hline (n) & $(3,550)$ & $(8,245)$ & \\
\hline \multicolumn{4}{|l|}{ Sexo } \\
\hline Hombre & $51.2(1,816)$ & $50.1(4,107)$ & \\
\hline Mujer & $48.8(1,734)$ & $49.9(4,138)$ & 0.513 \\
\hline \multicolumn{4}{|l|}{ Edad (años) } \\
\hline $12-13$ & $27.6(1,019)$ & $25.3(2,098)$ & 0.124 \\
\hline $14-15$ & $24.8(922)$ & $27.0(2,206)$ & \\
\hline $16-17$ & $25.3(848)$ & $24.7(2,018)$ & \\
\hline $18-19$ & $22.3(761)$ & $23.0(1,923)$ & \\
\hline Realiza AFD & $44.0(1,469)$ & $55.6(4,594)$ & $<0.001$ \\
\hline Vive con su padre & $80.8(2,869)$ & $75.2(6,198)$ & 0.001 \\
\hline Vive con su madre & $97.3(3,447)$ & $93.1(7,656)$ & $<0.001$ \\
\hline Vive con su padre y madre & $78.3(2,776)$ & $70.2(5,772)$ & $<0.001$ \\
\hline \multicolumn{4}{|l|}{ Localidad de residencia } \\
\hline Ciudades & $44.2(1,193)$ & $45.0(3,656)$ & 0.992 \\
\hline Urbanas & $15.3(762)$ & $14.3(1,148)$ & \\
\hline Mixtas & $14.6(372)$ & $14.7(1,203)$ & \\
\hline \multirow[t]{2}{*}{ Rurales } & $25.9(1,223)$ & $26.0(2,238)$ & \\
\hline & $\mathrm{x}$ & $\bar{x}$ & $\mathrm{p}$ \\
\hline $\begin{array}{l}\text { Tiempo reportado de AFD, } \\
\mathrm{min} / \mathrm{sem}\end{array}$ & 179.42 & 186.84 & 0.250 \\
\hline
\end{tabular}

$\mathrm{AFD}=$ actividad física deportiva; $\mathrm{Min} / \mathrm{sem}=$ minutos a la semana; $\overline{\mathrm{x}}$ = media.

aumentó la prevalencia de adolescentes que reportaron practicar AFD (44.0\% a 55.6\%), aunque no hubo diferencias sobre el tiempo que reportaron realizarlas. De 2002 a 2014 disminuyó la proporción de adolescentes que vivían con su padre, con su madre o con ambos. En este periodo de tiempo no hubo diferencias respecto a la distribución por tamaño de localidad.

En este lapso aumentó la proporción de padres o madres con educación media o superior, además de quienes reportaron realizar AFD (Tabla 2). No existieron diferencias entre los años en la distribución de la edad de los padres y madres.

En ambos años, en comparación con las niñas, fue más probable que los niños realizaran AFD y que reportaran dedicar más tiempo a realizar AFD (Tabla 3). A medida que aumentó la edad de los adolescentes, fue menos probable realizaran AFD y dedicaban menos tiempo a ésta, aunque este último patrón fue más claro en 2002 que en 2014. En ambos años, a medida que aumentó la escolaridad de los padres y madres, aumentó la probabilidad de que los adolescentes realizaran AFD y el tiempo destinado a ésta; sin embargo, las diferencias de acuerdo con el tiempo solo fueron significativas 
Tabla 2 - Características sociodemográficas y de práctica de actividad física deportiva en padres y madres de adolescentes mexicanos en 2002 y 2014

\begin{tabular}{|c|c|c|c|c|c|c|}
\hline & \multicolumn{2}{|c|}{ Padres } & \multicolumn{4}{|c|}{ Madres } \\
\hline & 2002 & 2014 & \multirow{3}{*}{$\mathrm{p}$} & 2002 & 2014 & \multirow{3}{*}{$\mathrm{p}$} \\
\hline & $\%(\mathrm{n})$ & $\%(\mathrm{n})$ & & $\%(\mathrm{n})$ & $\%(\mathrm{n})$ & \\
\hline & $100.0(2,588)$ & $100.0(5,573)$ & & $100.0(3,213)$ & $100.0(7,048)$ & \\
\hline \multicolumn{7}{|l|}{ Edad (años) } \\
\hline 20-30 & $2.5(79)$ & $3.9(209)$ & 0.240 & $4.3(146)$ & $3.4(241)$ & 0.428 \\
\hline $31-40$ & $33.0(877)$ & $31.0(1,724)$ & & $43.4(1,436)$ & $44.1(3,137)$ & \\
\hline $41-50$ & $44.6(1,127)$ & $46.9(2,631)$ & & $37.7(1,228)$ & $39.4(2,776)$ & \\
\hline $51-60$ & $19.9(505)$ & $18.2(1,009)$ & & $14.6(403)$ & 13.1 (894) & \\
\hline \multicolumn{7}{|l|}{ Escolaridad } \\
\hline Ninguno & $14.3(476)$ & $6.8(425)$ & $<0.001$ & $17.6(626)$ & $8.7(640)$ & $<0.001$ \\
\hline Primaria & $47.8(1,467)$ & $34.8(2,156)$ & & $51.0(1,832)$ & $36.2(2,746)$ & \\
\hline Secundaria & 19.9 (484) & $30.5(1,937)$ & & $21.8(663)$ & $33.9(2,712)$ & \\
\hline Preparatoria & $8.7(225)$ & $15.6(958)$ & & $5.2(185)$ & $12.5(935)$ & \\
\hline Superior & $9.3(217)$ & $12.3(722)$ & & $4.4(141)$ & $8.7(623)$ & \\
\hline Realiza AFD & $14.4(380)$ & $27.2(1,700)$ & $<0.001$ & $5.35(157)$ & $21.5(1,246)$ & $<0.001$ \\
\hline
\end{tabular}

$\mathrm{AFD}=$ actividad física deportiva

en 2014. En 2014 fue mayor la probabilidad de que los adolescentes realizaran AFD y dedicaran más tiempo a ésta a medida que aumentó la edad de sus padres. En ambos años, la probabilidad de que los adolescentes realizaran AFD fue mayor cuando sus padres y madres también la realizaban; además, en 2014 fue mayor el tiempo que los adolescentes destinaban a realizar AFD cuando sus padres o madres también la realizaban, en comparación con sus contrapartes. Únicamente en 2014 se observó que los adolescentes que residían en localidades rurales reportaron realizar menos tiempo AFD que aquellos que viven en ciudades.

En los tres modelos, respecto a 2002, en 2014 aumentó la probabilidad de que los adolescentes realizaran AFD (Tabla 4). En comparación con sus contrapartes, las mujeres y los adolescentes de mayor edad tuvieron menor probabilidad de realizar AFD. En el modelo 1 se observó que los escolares que tenían padres con educación secundaria o más, mayores de 31 años y que realizaran $\mathrm{AFD}$, tuvieron mayor probabilidad de realizar AFD. En el modelo 2 los adolescentes que tenían una madre con educación preparatoria o superior y que realizaran AFD, tuvieron mayor probabilidad de que practiquen AFD. En el modelo 3 se observó que la probabilidad de que los adolescentes realizaran AFD fue mayor cuando éstos tenían padres con educación superior, mayores de 41 años, que realizaran AFD, y con madres con educación preparatoria que realizaran AFD. En este modelo se observó que la práctica de AFD de los padres, en comparación con la de madres, aumentó en mayor medida la probabilidad de que adolescentes realizaran AFD.

En los modelos 1, 2 y 3 existieron interacciones entre el año de la encuesta con la AFD de los padres (RM $=0.50)$, de las madres $(\mathrm{RM}=0.47)$ y de los padres $(\mathrm{RM}=0.46)$, respectivamente; por lo cual las probabilidades de que los adolescentes realicen AFD de acuerdo con estas dos variables se graficaron en la Figura 1. En los tres modelos se mostró que la probabilidad de que los adolescentes realicen AFD fue mayor entre aquellos cuyos padres (Figura 1.a.), madres (Figura 1.b.) y padres (Figura 1.c.) realizaban AFD, respecto a los adolescentes cuyos padres o madres no realizaban AFD. Por ejemplo, en 2002 los adolescentes cuyo padre realizaba AFD tenían 2 veces más probabilidad de realizar AFD respecto a los adolescentes cuyos padres no realizaban AFD $(0.76 / 0.38=2.00)$ (Figura 1.c). Para el 2014 esta razón disminuyó a 1.74 veces (0.82 / 0.47 = 1.74). El mismo patrón se observó con las otras dos interacciones (Figuras 1.a y 1.b).

\section{Discusión}

En el presente estudio se observó que entre 2002 y 2014 más adolescentes mexicanos reportaron realizar AFD. En ese lapso aumentó la proporción de padres y madres con mayor escolaridad y que realizaban AFD. La prevalencia de AFD de adolescentes mexicanos disminuyó conforme aumentó la edad, pero fue mayor en hombres y en aquellos cuyos padres tenían mayor nivel educativo o realizaban AFD. 
Tabla 3 - Prevalencia de práctica de actividad física deportiva en adolescentes mexicanos de acuerdo con características sociodemográficas en 2002 y 2014

\begin{tabular}{|c|c|c|c|c|c|c|}
\hline & \multicolumn{3}{|c|}{2002} & \multicolumn{3}{|c|}{2014} \\
\hline Características & $\%$ & $\mathrm{p}$ & $\begin{array}{c}\text { Media } \\
\text { (minutos/ } \\
\text { semana) }\end{array}$ & $\%$ & $\mathrm{p}$ & $\begin{array}{c}\text { Media } \\
\text { (minutos/ } \\
\text { semana) }\end{array}$ \\
\hline \multicolumn{7}{|l|}{ Adolescentes } \\
\hline Total & 44.0 & & 179.4 & 55.6 & & 186.8 \\
\hline \multicolumn{7}{|l|}{ Sexo } \\
\hline Hombre & 59.3 & $<0.001$ & 247.2 & 70.6 & $<0.001$ & 261.4 \\
\hline Mujer & 27.9 & & $89.5^{\mathrm{a}}$ & 40.6 & & $107.9^{\mathrm{a}}$ \\
\hline \multicolumn{7}{|l|}{ Edad (años) } \\
\hline $12-13$ & 48.4 & 0.049 & 218.8 & 58.8 & $<0.001$ & 177.9 \\
\hline $14-15$ & 45.4 & & 185.5 & 59.2 & & 192.2 \\
\hline $16-17$ & 43.0 & & $138.7^{\mathrm{a}}$ & 56.6 & & $211.2^{\mathrm{a}}$ \\
\hline $18-19$ & 38.1 & & $128.6^{a}$ & 46.8 & & $155.6^{\mathrm{bc}}$ \\
\hline \multicolumn{7}{|l|}{ Padres } \\
\hline \multicolumn{7}{|l|}{ Escolaridad } \\
\hline Ninguno & 37.2 & $<0.001$ & 162.3 & 45.4 & $<0.001$ & 133.7 \\
\hline Primaria & 41.5 & & 167.3 & 54.7 & & $179.6^{a}$ \\
\hline Secundaria & 45.6 & & 189.1 & 52.8 & & 166.9 \\
\hline Preparatoria & 37.9 & & 133.2 & 59.3 & & $207.7^{\mathrm{ac}}$ \\
\hline Superior & 68.6 & & 226.4 & 68.3 & & $265.1^{\text {abcd }}$ \\
\hline \multicolumn{7}{|l|}{ Edad (años) } \\
\hline $20-30$ & 24.1 & 0.104 & 91.3 & 35.6 & $<0.001$ & 91.2 \\
\hline $31-40$ & 42.5 & & 172.4 & 55.1 & & $173.6^{\mathrm{a}}$ \\
\hline $41-50$ & 47.4 & & $186.3^{\mathrm{a}}$ & 57.8 & & $200.1^{a}$ \\
\hline $51-60$ & 45.8 & & 180.6 & 57.3 & & $202.6^{a}$ \\
\hline \multicolumn{7}{|l|}{ Realiza AFD } \\
\hline No & 39.8 & $<0.001$ & 161.7 & 52.5 & $<0.001$ & 171.3 \\
\hline Sí & 68.2 & & 242.6 & 65.0 & & $230.1^{a}$ \\
\hline \multicolumn{7}{|l|}{ Madres } \\
\hline \multicolumn{7}{|l|}{ Escolaridad } \\
\hline Ninguno & 41.2 & 0.044 & 174.3 & 48.9 & $<0.001$ & 156.5 \\
\hline Primaria & 41.2 & & 160.7 & 53.6 & & 170.5 \\
\hline Secundaria & 47.5 & & 179.8 & 53.6 & & 178.1 \\
\hline Preparatoria & 55.5 & & 185.0 & 63.0 & & $209.7^{\mathrm{ab}}$ \\
\hline Superior & 58.7 & & 210.4 & 68.9 & & $272.3^{\text {abcd }}$ \\
\hline \multicolumn{7}{|l|}{ Edad (años) } \\
\hline $20-30$ & 43.5 & 0.567 & 199.6 & 50.6 & 0.388 & 146.0 \\
\hline $31-40$ & 43.9 & & 174.3 & 56.3 & & 177.2 \\
\hline $41-50$ & 47.3 & & 180.1 & 57.1 & & $198.6^{a}$ \\
\hline $51-60$ & 41.8 & & 148.0 & 55.9 & & 193.4 \\
\hline \multicolumn{7}{|l|}{ Realiza AFD } \\
\hline No & 42.2 & $<0.001$ & 173.0 & 53.0 & $<0.001$ & 174.3 \\
\hline Sí & 70.8 & & 175.9 & 67.0 & & $236.5^{\mathrm{a}}$ \\
\hline \multicolumn{7}{|l|}{ Localidad } \\
\hline Ciudades & 47.6 & 0.148 & 172.5 & 56.2 & 0.231 & 194.2 \\
\hline Urbanas & 43.6 & & 193.0 & 57.3 & & 194.6 \\
\hline Mixtas & 44.1 & & 138.5 & 56.5 & & 183.6 \\
\hline Rurales & 37.9 & & 170.6 & 53.1 & & $164.0^{\mathrm{a}}$ \\
\hline
\end{tabular}

$\mathrm{AFD}=$ actividad física deportiva; $\mathrm{a}=$ diferencia significativa en comparación con la primer categoría; $\mathrm{b}=$ diferencia significativa en comparación con la segunda categoría; $\mathrm{c}=$ diferencia significativa en comparación con la tercer categoría; $\mathrm{d}$ = diferencia significativa en comparación con la cuarta categoría. Los superíndices indican que los intervalos de confianza al $95 \%$ no se traslapan.

La prevalencia de adolescentes mexicanos que reportó realizar AFD aumentó $11.6 \%$ en 12 años, además de que poco más de la mitad reportó realizar AFD en 2014. Según los resultados de la Encuesta Nacional de Salud y Nutrición, entre 2012 y 2016, ${ }^{6}$ más de la mitad de los adolescentes mexicanos son activos físicamente, sin embargo, parece ser que actualmente los adolescentes están siendo menos activos. En 2006 el 69.6\% de adolescentes mexicanos fueron clasificados como activos, esta prevalencia aumentó para 2012 (77.7\%) pero disminuyó en 2016 (60.5\%). Con este grupo etario se indagó sobre las actividades realizadas los 12 meses previos de la aplicación de la encuesta, ${ }^{14}$ diferente al presente documento donde se preguntó sobre las actividades realizadas la semana previa de la aplicación de la encuesta; probablemente por esta razón exista una diferencia entre las prevalencias de la población adolescente mexicana activa en ambas encuestas ( 10\%).

Generalmente los términos de AF y ejercicio se usan de forma indistinta, del mismo modo que los de AF y deporte, sin embargo, no son sinónimos. El hecho de que en la ENUT y en la ENSANUT se indague en la misma pregunta sobre actividades físicas moderadas o vigorosas (e.g. caminar o trotar) y actividades deportivas (e.g. fútbol), además de que se reporte la sumatoria del tiempo destinado a estas actividades, provoca que se refuerce el uso incorrecto de los términos y que las estadísticas posiblemente estén sesgadas, por lo que se debe de considerar diferenciar en la terminología y preguntar sobre estos comportamientos de forma individual ya que las prevalencias pueden ser disímiles. En un estudio con adolescentes mexicanos, ${ }^{15}$ la prevalencia de adolescentes activos fue disímil cuando se diferenció entre actividad física y la pertenencia a un equipo deportivo (87.2\% y $43.7 \%$, respectivamente).

En ambos años los niños fueron más activos que las niñas, coincidiendo con lo reportado en diferentes muestras de adolescentes ${ }^{16}$. Esto puede deberse a estereotipos de género relacionados a la AFD donde a los niños se les inculca mayor participación en actividades competitivas (i.e. deportivas), mientras que a las niñas en actividades más tranquilas.

La práctica de AFD disminuye a medida que aumenta la edad, lo anterior es una tendencia sistemática en el pre- 
Tabla 4 - Modelos de regresión logística donde la variable dependiente es la práctica de actividad física deportiva de adolescentes y las variables independientes son las características sociodemográficas

\begin{tabular}{|c|c|c|c|c|c|c|}
\hline & \multicolumn{2}{|c|}{ Modelo 1} & \multicolumn{2}{|c|}{ Modelo 2} & \multicolumn{2}{|c|}{ Modelo 3} \\
\hline & $\mathrm{RM}$ & IC95\% & $\mathrm{RM}$ & IC95\% & $\mathrm{RM}$ & IC95\% \\
\hline \multicolumn{7}{|l|}{ Año } \\
\hline 2002 & 1.00 & & 1.00 & & 1.00 & \\
\hline 2014 & 1.61 & $1.32-1.96$ & 1.48 & $1.21-1.81$ & 1.52 & $1.24-1.86$ \\
\hline \multicolumn{7}{|l|}{ Sexo } \\
\hline Varones & 1.00 & & 1.00 & & 1.00 & \\
\hline Mujer & 0.26 & $0.22-0.30$ & 0.25 & $0.22-0.30$ & 0.25 & $0.22-0.30$ \\
\hline \multicolumn{7}{|l|}{ Edad (años) } \\
\hline $12-13$ & 1.00 & & 1.00 & & 1.00 & \\
\hline $14-15$ & 0.88 & $0.71-1.08$ & 0.90 & $0.74-1.10$ & 0.89 & $0.72-1.10$ \\
\hline $16-17$ & 0.73 & $0.61-0.88$ & 0.82 & $0.67-1.00$ & 0.75 & $0.62-0.92$ \\
\hline $18-19$ & 0.53 & $0.42-0.67$ & 0.56 & $0.45-0.70$ & 0.54 & $0.42-0.69$ \\
\hline \multicolumn{7}{|l|}{ Escolaridad (padre) } \\
\hline Ninguna & 1.00 & & & & 1.00 & \\
\hline Primaria & 1.38 & $0.97-1.96$ & & & 1.38 & $0.96-1.97$ \\
\hline Secundaria & 1.49 & $1.01-2.19$ & & & 1.37 & $0.90-2.08$ \\
\hline Preparatoria & 1.54 & $1.03-2.31$ & & & 1.23 & $0.80-1.88$ \\
\hline Superior & 3.09 & $1.89-5.06$ & & & 2.30 & $1.31-4.02$ \\
\hline \multicolumn{7}{|l|}{ Edad del padre (años) } \\
\hline $20-30$ & 1.00 & & 1.00 & & 1.00 & \\
\hline $31-40$ & 1.87 & $1.28-2.74$ & & & 1.63 & $0.92-2.89$ \\
\hline $41-50$ & 2.08 & $1.43-3.02$ & & & 2.01 & $1.10-3.68$ \\
\hline $51-60$ & 2.31 & $1.51-3.53$ & & & 2.08 & $1.10-3.93$ \\
\hline \multicolumn{7}{|l|}{ AFD (padre) } \\
\hline No realiza & 1.00 & & & & 1.00 & \\
\hline Sí realiza & 6.40 & $2.33-17.54$ & & & 6.90 & $2.54-18.75$ \\
\hline Año * AFD (padre) & 0.50 & $0.30-0.84$ & & & 0.46 & $0.27-0.77$ \\
\hline \multicolumn{7}{|l|}{ Escolaridad (madre) } \\
\hline Ninguna & & & 1.00 & & 1.00 & \\
\hline Primaria & & & 1.04 & $0.77-1.42$ & 0.93 & $0.68-1.28$ \\
\hline Secundaria & & & 1.32 & $0.92-1.88$ & 1.12 & $0.79-1.60$ \\
\hline Preparatoria & & & 2.05 & $1.37-3.06$ & 1.54 & $1.03-2.29$ \\
\hline Superior & & & 2.44 & $1.50-3.98$ & 1.41 & $0.85-2.35$ \\
\hline \multicolumn{7}{|l|}{ Edad de la madre (años) } \\
\hline $20-30$ & & & 1.00 & & 1.00 & \\
\hline $31-40$ & & & 0.95 & $0.65-1.37$ & 0.76 & $0.51-1.15$ \\
\hline $41-50$ & & & 1.06 & $0.73-1.54$ & 0.70 & $0.45-1.08$ \\
\hline $51-60$ & & & 1.12 & $0.75-1.69$ & 0.86 & $0.51-1.46$ \\
\hline \multicolumn{7}{|l|}{ AFD (madre) } \\
\hline No realiza & & & 1.00 & & 1.00 & \\
\hline Sí realiza & & & 6.82 & $1.64-28.29$ & 1.59 & $1.27-1.97$ \\
\hline Año* AFD (madre) & & & 0.47 & $0.23-0.98$ & & \\
\hline
\end{tabular}

$\mathrm{RM}$ = razón de momios; IC95\% = intervalo de confianza al 95\%; AFD = actividad física deportiva; Año, año de la encuesta; AFD = actividad física deportiva. Interacción: Producto del año de la encuesta con la actividad física deportiva del padre o madre. Modelo 1 = Ajustado por año de la encuesta, sexo (adolescente), edad (adolescente), escolaridad (padre), práctica deportiva (padre). Modelo 2 = Ajustado por año de la encuesta, sexo (adolescente), edad (adolescente), escolaridad (madre), práctica deportiva (madre). Modelo 3 = Ajustado por año de la encuesta, sexo (adolescente), edad (adolescente), escolaridad (padre y madre), práctica deportiva (padre y madre).

sente análisis y en estudios realizados con adolescentes ${ }^{16}$. ye la práctica de AFD conforme aumenta la edad son: a) Las principales razones por las que se cree que disminu- insatisfacción de las clases de educación física: en la ado- 
1a - Interacción entre la práctica deportiva del padre con el año de la encuesta

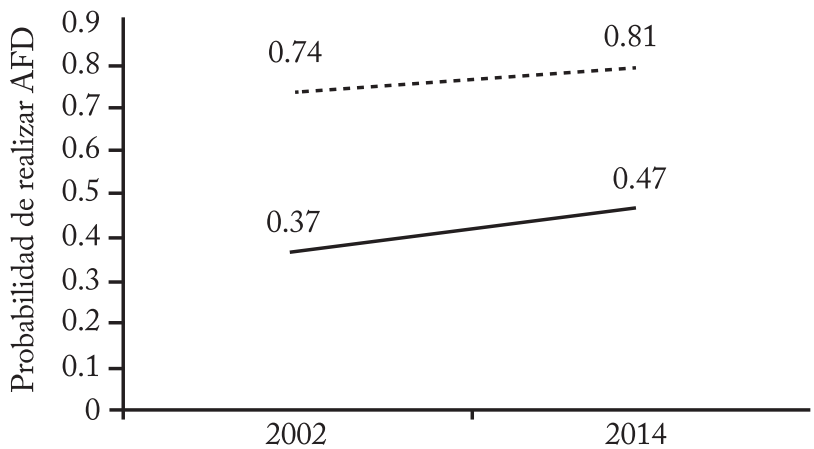

$1 \mathrm{~b}$ - Interacción entre la práctica deportiva de la madre con el año de la encuesta

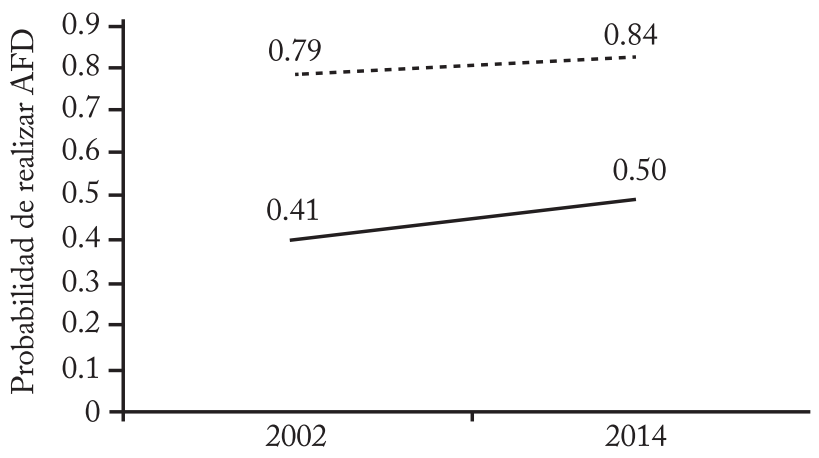

1c - Interacción entre la práctica deportiva del padre y de la madre con el año de la encuesta

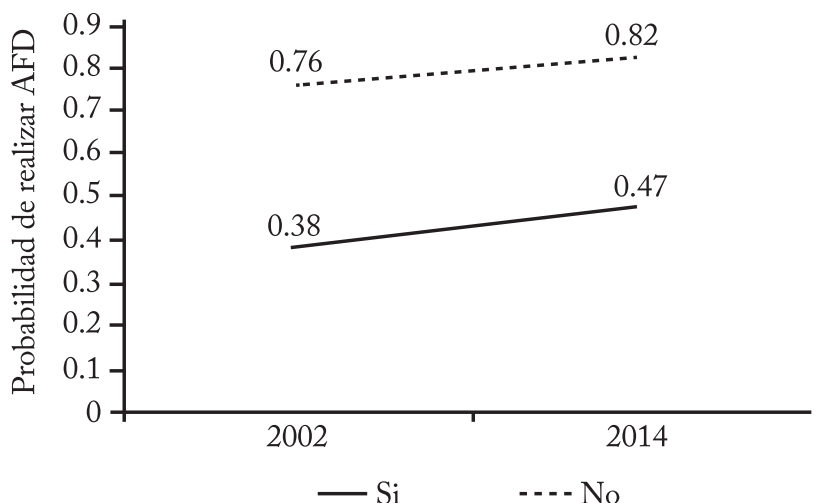

Figura 1 - Interacciones entre la práctica de actividad física deportiva de padres y/o madres con el año de la encuesta para predecir la práctica de actividad física deportiva de adolescentes mexicanos $\mathrm{AFD}=$ actividad física deportiva

lescencia las clases pueden ser percibidas como aburridas principalmente cuando duran más de 50 minutos $^{17}$; b) actividades durante el tiempo libre: actualmente existen diferentes alternativas para el tiempo libre (e.g. televisión, computadora, videojuegos y celulares) ${ }^{18}$; c) competición: el modelo de deporte escolar se basa generalmente en la competitividad y el éxito competitivo, lo cual puede desalentar la participación de la práctica de $\mathrm{AFD}^{19}$; d) exigencia escolar: ésta se incrementa conforme aumenta la edad y por tanto se dispone de menos tiempo libre ${ }^{19,20}$.

En ambos años se mostró que a medida que aumentó la escolaridad de los padres y madres (i.e. un indicador del nivel socioeconómico) fue mayor la probabilidad de que los adolescentes reportaran realizar AFD. Se ha observado esta misma tendencia en países industrializados ${ }^{21}$, pero es escasa la evidencia en países de ingreso medio ${ }^{22}$. En un estudio con una muestra reducida de adolescentes norteamericanos ${ }^{23}$ se reportó que cuando el nivel socioeconómico es menor, disminuye la probabilidad de realizar deporte y AFMV. En el caso de México, más de la mitad de las comunidades no cuentan con espacios adecuados para realizar actividad física ${ }^{7}$. Además del nivel socioeconómico, la práctica de ciertos tipos de $\mathrm{AF}$ es menor cuando se reside a mayor distancia de infraestructura deportiva ${ }^{24}$. Por lo tanto, posiblemente las diferencias en el reporte de la práctica de AFD puedan deberse a que cuando existe mayor nivel socioeconómico es más probable que las personas tengan acceso a la práctica deportiva (e.g. residir cerca de instalaciones deportivas públicas, pertenecer a un club deportivo, así como poder pagar cuotas de entrenamiento, uniformes, torneos, etc.). Del mismo modo cuando las personas tienen mayor nivel educativo posiblemente tengan mayor conciencia sobre la importancia de tener un estilo de vida saludable, lo cual fomente la práctica de AFD.

No se encontraron estudios que evalúen la relación de la edad de los padres y/o madres con la práctica de AFD de los adolescentes. Se esperaba que los padres más jóvenes promovieran más la AFD; sin embargo, se encontró lo contrario. Esto podría deberse a que los adolescentes en estudio provenían de hogares "consolidados" donde los padres son mayores a 40 años y cuyos hijos menores tienen 13 años o más. El vivir en este tipo de hogares permite que los adolescentes tengan mejores condiciones de vida, pues generalmente cuentan con economías más sólidas permitiendo mayor accesibilidad a la práctica de $\mathrm{AFD}^{25}$. Otra posible explicación es que los padres de mayor edad y que viven en un hogar consolidado, cuentan con mayor experiencia en la crianza por lo que inculcan hábitos saludables a sus hijos más jóvenes.

Aunque la asociación de la AFD de los padres con la AFD de los adolescentes ha sido un hallazgo consisten$\mathrm{te}^{9,26}$, nuestro análisis evidenció dos patrones nuevos. E1 primero es que no siempre la influencia determinante viene dada por ambos padres ya que en nuestro estudio se observó que cuando los adolescentes viven con ambos padres, la práctica de AFD reportada por el padre 
influye más en la práctica de AFD de los adolescentes que la AFD reportada por la madre. En contraste, en estudios previos se había observado que la AFD de ambos padres tenía efectos sobre la AFD de adolescentes $^{27}$ o bien la influencia de la madre es mayor ${ }^{9}$. Sólo en una muestra de adolescentes españoles ${ }^{27}$ los padres influyeron más que las madres. Se debe señalar que en los estudios previos no se diferenció entre adolescentes de familias nucleares de los de familias monoparentales. Posiblemente las madres que realizan ejercicio buscan un "espacio libre", donde puedan olvidarse de la crianza y labores del hogar, dedicando tiempo en su persona y/o socializar; mientras que los padres pueden aprovechar estos espacios para convivir con sus hijos ya que es más probable que sean jefes del hogar y dediquen más tiempo a trabajar que a convivir con sus familias. En el presente estudio se observó que una proporción importante de los adolescentes no vivían con ambos padres. El segundo hallazgo relevante es que la influencia de la AFD reportada por los padres sobre la AFD reportada por los adolescentes disminuyó en el periodo bajo estudio. En estudios previos se ha reportado que en poblaciones pediátricas los amigos ${ }^{28}$ y los profesores de educación física ${ }^{29}$ tienen influencia en la práctica de AF. Probablemente los amigos y los profesores de educación física han comenzado a influir en mayor medida sobre la práctica de AFD de los adolescentes ya que éstos pasan gran parte del día en la escuela.

La principal fortaleza de esta investigación es que, hasta nuestro conocimiento, este es el primer trabajo en una muestra representativa de adolescentes de un país de ingreso medio, donde se analiza la evolución en el tiempo de la prevalencia de AFD, así como la influencia en la práctica deportiva de características sociodemográficas de adolescentes y sus padres y/o madres.

Las limitaciones del presente análisis son que la ENUT no tenía como finalidad analizar los cambios en la prevalencia de AFD en adolescentes mexicanos, por lo que las variables con las que se cuenta para analizar los patrones en AFD son limitadas. Además, se preguntó respecto a la práctica de deporte o ejercicio, sin especificar la diferencia entre éstos, por lo que probablemente los encuestados presenten dificultades para diferenciar entre estos comportamientos y subsecuentemente contestar adecuadamente esta pregunta. Se recomienda realizar un estudio similar, con una muestra representativa de la población pediátrica y adulta mexicana donde se modifique el apartado de AFD de modo que se diferencie entre AFMV, ejercicio y deporte (i.e. recreativo o competitivo) además de que se cuente con variables que puedan aportar información más detallada (e.g. tipo de actividad, experiencia en la actividad, frecuencia, duración, intensidad y/o diferenciar en si se realiza por ocio, educación, salud o competencia).

En síntesis, en el transcurso de 12 años aumentó la prevalencia de AFD reportada por adolescentes mexicanos. El sexo, la edad, tener un padre y/o madre con mayor nivel educativo y que realice AFD son variables asociadas con la AFD de adolescentes mexicanos. Se requiere de políticas y programas públicos diseñados a la promoción de estilos de vida activos enfocados a adolescentes y a sus padres o madres, donde se centre principal atención en los grupos que presentan menor probabilidad de realizar AFD.

\section{Conflicto de intereses}

Los autores declaran que no hay conflicto de intereses.

\section{Financiamiento}

No existieron fuentes de financiamiento para la elaboración del manuscrito.

\section{Contribuição dos autores}

Ayala-Guzmán CI participó en el análisis e interpretación de datos, búsqueda de literatura y en la edición y aprobación final del artículo. Ayala-Hilario C participó en el análisis de los datos, búsqueda de literatura y elaboración del manuscrito y aprobación final del mismo. Ortiz-Hernández L fue responsable de la concepción del estudio, supervisión del análisis de datos y la edición y aprobación final del artículo.

\section{Agradecimientos}

Al Lic. Nutr. Christian Jesús Gascón Sánchez y a la Lic. Nutr. Carolina Alvarenga Dantas de Lemos por su apoyo con la revisión del resumen en portugués.

\section{Referencias}

1. American College of Sports Medicine. ACSM's Guidelines for Exercise Testing and Prescritpion. Exercise Prescription for Healthy Populations with Special Considerations. Children and adolecents. In: Riebe D, Ehrman JK, Liguori G, Magal M, editors. 10th ed. Philadelphia. 2018:180-083.

2. FAO Food and nutrition technical report series. Human energy requirements. Report of a Joint FAO/WHO/UNU Expert Consultation. Roma: United Nations University, World Health Organization, Food and Agriculture Organization of the United Nations; 2004. Disponible en: http://www.fao.org/3/y5686e/y5686e00.htm

3. Nelson TF, Stovitz SD, Thomas M, LaVoi NM, Bauer KW, Neumark-Sztainer D. Do youth sports prevent pediatric obesity? A systematic review and commentary. Curr Sports Med Rep. 2011;10(6):360-70. 
4. Drake KM, Beach ML, Longacre MR, MacKenzie T, Titus LJ, Rundle AG, et al. Influence of Sports, Physical Education, and Active Commuting to School on Adolescent Weight Status. Pediatrics. 2012;130(2):e296-e304.

5. Knuth AG, Hallal PC. Temporal trends in physical activity: a systematic review. J Phys Act Health. 2009;6(5):548-59.

6. Hernández MA, Rivera JD, Shamah TS, Cuevas L, GómezAcosta LM, Gaona-Pineda EB. Encuesta Nacional de Salud y Nutrición de Medio Camino 2016. (ENSANUT MC 2016). Informe final de resultados. Cuernavaca, México: Instituto Nacional de Salud Pública de México; 2016.

7. Galaviz KI, García GA, Gaytán-González A, GonzalezCasanova I, Villalobos MFG, Jauregui A, et al. Results from Mexico's 2018 Report Card on Physical Activity for Children and Youth. J Phys Act Health. 2018;15(2):S384-S5.

8. Pfeffer F, Perez-Rodriguez M, Meléndez G, Gámez E, Loria A, Hernández CI, et al. Physical Activity Patterns in Mexican School-Aged Children. J Obes Weight Loss Ther. 2014;4(2):2-5.

9. Schoeppe S, Vandelanotte C, Bere E, Lien N, Verloigne $\mathrm{M}$, Kovacs $\mathrm{E}$, et al. The influence of parental modelling on children's physical activity and screen time: Does it differ by gender? Eur J Public Health. 2017;27(1):152-7.

10. Jin Y, Jones-Smith JC. Associations Between Family Income and Children's Physical Fitness and Obesity in California, 2010-2012. Prev Chronic Dis.2015;12:140392.

11. Vilhjalmsson R, Kristjansdottir G. Gender differences in physical activity in older children and adolescents: the central role of organized sport. Soc Sci Med. 2003;56(2):363-74.

12. Dumith SC, Gigante DP, Domingues MR, Kohl HW, 3rd. Physical activity change during adolescence: a systematic review and a pooled analysis. Int J Epidemiol. 2011;40(3):685-98.

13. Instituto Nacional de Estadística y geografía [INEGI]. Encuesta Nacional sobre Uso del Tiempo. Available from: https:/www.inegi.org.mx/programas/enut/2014/default.html

14. Craig C, Marshall A, Sjostrom M, Bauman A, Booth $\mathrm{M}$, Ainsworth B, et al. International physical activity questionnaire: 12-country reliability and validity. Med Sci Sports Exerc. 2003;35(8):1381-95.

15. Hidalgo-Rasmussen CA, Ramírez-López G, HidalgoSan Martín A. Actividad física, conductas sedentarias y calidad de vida en adolescentes universitarios de Ciudad Guzmán, Jalisco, México. Ciênc. saúde coletiva [online]. 2013;18(7):1943-52.

16. Craggs $C$, Corder K, van Sluijs EMF, Griffin SJ. Determinants of change in physical activity in children and adolescents: a systematic review. Am J Prev Med. 2011;40(6):645-58

17. Barroso G. El área de educación física y su influencia en la adquisición de hábitos saludables. Granada, España: Universidad de Granada; 2007.

18. Sánchez Pardo L, Crespo Herrador G, Aguilar-Moya R, Bueno-Cañigral F, Aleixandre-Benavent R, ValderramaZurián J-C. Los adolescentes y las tecnologías de la información y la comunicación (TIC). 2015.
19. Nuviala AN, Juan FR, Montes MEG. Tiempo libre, ocio y actividad física en los adolescentes: La influencia de los padres. Retos. 2003;6:13-20.

20. Martínez Baena AC, Chillón P, Martín-Matillas M, Pérez López I, Castillo R, Zapatera B, et al. Motivos de abandono y no práctica de actividad físico-deportiva en adolescentes españoles: estudio Avena. Cuadernos de Psicología del Deporte. 2012;12(1):45-54.

21. McCormack LA, Meendering J. Diet and Physical Activity in Rural vs Urban Children and Adolescents in the United States: A Narrative Review. J Acad Nutr Diet. 2016;116(3):467-80.

22. Strong LL, Anderson CB, Miranda PY, Bondy ML, Zhou R, Etzel C, et al. Gender differences in sociodemographic and behavioral influences of physical activity in Mexican-origin adolescents. J Phys Act Health. 2012;9(6):829-39.

23. Kwon S, Janz KF, Letuchy EM, Burns TL, Levy SM. Parental characteristic patterns associated with maintaining healthy physical activity behavior during childhood and adolescence. Int J Behav Nutr Phys Act. 2016;13(1):58.

24. Eime RM, Charity MJ, Harvey JT, Payne WR. Participation in sport and physical activity: associations with socioeconomic status and geographical remoteness. BMC Public Health. 2015;15:434.

25. Paredes PB. Capítulo IV. Hogares en consolidación. Descripción y análisis de un equilibrio precario. In: González-Rocha M, Agudo AS, Castañeda AV, Paredes PB, Triano ME, Villagómez PO, (editors). Procesos Domésticos y Vulnerabilidad Perspectivas antropológicas de los hogares con Oportunidades. 1ra ed. México, D.F.: Centro de Investigaciones y Estudios Superiores en Antropología Social (CIESAS); 2006. p. 237.

26. Edwardson CL, Gorely T. Parental influences on different types and intensities of physical activity in youth: A systematic review. Psychol Sport Exerc. 2010;11(6):522-35.

27. Martín MAG, Rebollo RM, Gaspar GC, Castro AMR, Perea JS, Rivero GG. Influencia de los hábitos de alimentación y actividad física de los padres y madres en sus hijos/as adolescentes. Sevilla, España: Ayuntamiento de Sevilla; Servicio de Salud; Observatorio de Salud; 2012.

28. Maturo CC, Cunningham SA. Influence of friends on children's physical activity: a review. Am J Public Health. 2013;103(7):e23-e38.

29. Bronikowski M, Bronikowska M, Laudańska-Krzemińska I, Kantanista A, Morina B, Vehapi S. PE Teacher and Classmate Support in Level of Physical Activity: The Role of Sex and BMI Status in Adolescents from Kosovo. Biomed Res Int. 2015;2015:290349.

Recibido: $16 / 05 / 2019$ Aprobado: 11/02/2020 